\title{
Video Article \\ Intravital Microscopy of Tumor-associated Vasculature Using Advanced Dorsal Skinfold Window Chambers on Transgenic Fluorescent Mice
}

\author{
Ann L.B. Seynhaeve ${ }^{1}$, Timo L.M. ten Hagen ${ }^{1}$ \\ ${ }^{1}$ Laboratory Experimental Surgical Oncology, Section Surgical Oncology, Department of Surgery, Erasmus MC
}

Correspondence to: Timo L.M. ten Hagen at t.I.m.tenhagen@erasmusmc.nl

URL: https://www.jove.com/video/55115

DOI: doi: $10.3791 / 55115$

Keywords: Medicine, Issue 131, Intravital microscopy, imaging, tumor, vasculature, transgenic mice, fluorescence

Date Published: $1 / 19 / 2018$

Citation: Seynhaeve, A.L., ten Hagen, T.L. Intravital Microscopy of Tumor-associated Vasculature Using Advanced Dorsal Skinfold Window Chambers on Transgenic Fluorescent Mice. J. Vis. Exp. (131), e55115, doi:10.3791/55115 (2018).

\section{Abstract}

Tumor and tumor vessel development, as well as tumor response to therapeutics, are highly dynamic biological processes. Histology provides static information and is often not sufficient for a correct interpretation. Intravital evaluation, in which a process is followed in time, provides extra and often unexpected information. With the creation of transgenic animals expressing cell-specific markers and live cell tracers, improvements to imaging equipment, and the development of several imaging chambers, intravital microscopy has become an important tool to better understand biological processes. This paper describes an experimental design for the investigation of tumor vessel development and of therapeutic effects in a spatial and temporal manner. Using this setup, the stage of vessel development, tip cell and lumen formation, blood flow, extravasation, an established vascular bed, and vascular destruction can be visualized and followed. Furthermore, therapeutic effects, intratumoral fate, and the localization of chemotherapeutic compounds can also be followed.

\section{Video Link}

The video component of this article can be found at https://www.jove.com/video/55115/

\section{Introduction}

While in vitro studies enable high-resolution kinetic imaging of processes, in vitro experimentation does not enable evaluation in the proper context. For instance, the interaction of tumor cells with stromal compartments or drug delivery and distribution inside a tumor cannot be studied in a culture plate. Animal models are therefore used to mimic the human physiology and pathology. However, the longitudinal imaging of processes, especially at a subcellular resolution, is challenging. Molecular imaging methods, such as magnetic resonance imaging (MRI), singlephoton emission computed tomography (SPECT), and positron emission tomography (PET), have good penetration depths but lack resolution or fail to illustrate anatomical structures. Optical imaging provides high resolution and enables the imaging of structures, but it is accompanied by poor to minimal penetration ${ }^{1}$. The application of intravital microscopy in combination with window chamber technologies, such as a dorsal skinfold or abdominal window, allows for high-resolution imaging in vivo ${ }^{2,3,4}$. This technology has distinct advantages, as it allows for longitudinal imaging over hours and even days, for imaging of processes in the proper context (e.g., cell-cell interactions in the imaged tissue), and for resolutions at the optical limits of advanced confocal and multiphoton microscopes.

The introduction of transgenic animals with cell- or protein-specific fluorescent labels opens a plethora of possibilities for in vivo and ex vivo experimentations. For instance, cell-cell interactions, the production of proteins, and the response to manipulation or therapy can be studied in vivo using these models ${ }^{5,6,7,8}$. Importantly, position in place and time can be determined with the proper imaging equipment and methodology. Here, the intravital microscopy of animals expressing an endothelial marker in combination with injectable agents in a tumor implanted in a modified dorsal skinfold window chamber is presented.

\section{Protocol}

All animal experiments were done in accordance with Dutch law, and protocols were approved by the Committee of Animal Experimentation of the Erasmus MC, Rotterdam, the Netherlands.

\section{Recipient Mouse}

1. When the transgenic mice are born, screen the animals for the appropriate genotype using standard procedures ${ }^{9}$. NOTE: In this manuscript, data obtained from an eNOStag-GFP line ${ }^{9}$ developed in-house and a purchased ROSA-mTmG (stock 007676) ${ }^{10}$ line are presented.

2. Use mice that are 12 weeks old or older and that are above $20 \mathrm{~g}$. 


\section{Donor Mouse}

NOTE: A tumor fragment for implantation in the dorsal window is obtained from a non-transgenic donor animal. Depending on the tumor type, normal (with syngeneic tumors) or immunodeficient (xenografts) mice are used.

1. Grow the tumor cells in a medium with the appropriate supplements in culture flasks at $37{ }^{\circ} \mathrm{C}$ and $5 \% \mathrm{CO}_{2}$.

2. Remove the medium from the cell flasks, wash once with $1 \times$ PBS, and detach the cells using $0.25 \%$ trypsin

3. Inactivate the trypsin by adding cell culture medium. Collect the cells, spin down at $1,200 \times \mathrm{g}$ for $5 \mathrm{~min}$, and resuspend the pellet in $5 \mathrm{~mL}$ of PBS.

4. Dilute $20 \mu \mathrm{L}$ of the cell suspension with $20 \mu \mathrm{L}$ of trypan blue, which stains dead cells. Count the number of living and dead cells using a hemocytometer; the number of dead cells should not exceed $10 \%$.

5. Spin the cells again at $1,200 \times \mathrm{g}$ for $5 \mathrm{~min}$ and resuspend the cell pellet in ice-cold PBS, yielding 1 million cells per $100 \mu \mathrm{L}$. Transport the cells on ice to the operating room.

6. Anesthetize the animal using isoflurane $/ \mathrm{O}_{2}$. Turn on the oxygen and adjust the flow to $0.5 \mathrm{~mL} / \mathrm{min}$. Adjust the isoflurane vaporizer to $3 \%$. After a few minutes, place the mouse in the anesthesia chamber. NOTE: Prefill the chamber to ensure it is ready for use to minimize stress.

7. When the mouse is sedated, bring the animal to the operating heating table, kept at $37^{\circ} \mathrm{C}$, and place the snout in the anesthesia nose cone.

1. NOTE: The animal is properly sedated when no reaction to a footpad pinch is noted.

8. Shave the mouse to better visualize the injection site. Inoculate using a $0.5 \mathrm{~mL}$ insulin syringe; inject $100 \mu \mathrm{L}$ of cells into the flank of the mouse and allow a tumor to develop.

9. Euthanize the animal by cervical dislocation under anesthesia as approved by your Institutional Animal Care and Use Committee. Using micro scissors and forceps, cut open the skin at the location of the tumor and dissect the tumor. Put the tumor in a Petri dish with PBS. Using micro scissors and forceps, cut the tumor into fragments of approximately $1 \mathrm{~mm}^{3}$ (Figure 1A-a).

NOTE: The tumor of the donor mouse should be big enough to give sufficient material, but large, necrotic tumors should be avoided. For most tumors, an average diameter of $10 \mathrm{~mm}$ suffices.

\section{Implantation of the Window Chamber}

1. Perform all procedures under aseptic conditions. Autoclave the surgical instruments (Figure 1A) and chamber materials (Figure 1B) NOTE: The window chamber used here, with a total weight of $1.1 \mathrm{~g}$, is made of polyether ether ketone (PEEK), a light, synthetic material that is MRI compatible. PEEK is 3.4-fold lighter than titanium, which is commonly used in the literature for these windows. It is resistant to most chemicals, is inert, does not provoke immune reactions, and is sturdy. This window is also smaller in design compared to previously published windows. Mice fitted with this window show full capacity of motion, can climb, and gain weight comparably to mice without a window chamber. Because the animal can bite on the window, synthetic windows may last a little shorter compared to titanium windows; however, they are easy to make and are inexpensive. These particular windows are made in-house and can be acquired upon request.

2. Sedate the recipient mouse using inhalation anesthetics (i.e., isoflurane/ $\mathrm{O}_{2}$ ) in an anesthesia chamber. Bring the mouse to the heated operating table kept at $37^{\circ} \mathrm{C}$ and place the snout in the anesthesia nose cone (see step 2.6). Apply eye ointment to avoid dryness.

3. Remove the hair from the entire back by shaving and applying hair removal gel. Take care that all gel is removed by rinsing the animal carefully with hand-warm tap water. Dry the animal and clean the skin with $70 \%$ ethanol.

NOTE: The shaving procedure can also be done the day before window implantation. The cream need to be removed thoroughly with handwarm water as this can cause skin irritation.

4. Mark the center line on the mouse spine with a marker to ensure the symmetrical positioning of chamber. Reposition the mouse, pull the skin into a flap using the center line as a folding position, and grasp the middle of flap. Position the window such that the top of the frame is at least $2 \mathrm{~mm}$ below the center line and circle the window view area on the skin with a marker.

5. Using a scalpel holder/blade size of 15 and micro scissors, remove the skin along the drawn circular line. Be careful not to damage underlying fascia.

NOTE: This creates the window view area of the chamber in which the tumor is transplanted.

6. Pull up the skinfold and use an ear puncher to punch holes $1 \mathrm{~mm}$ in diameter through the skin, one on either side of the view area (Figure $1 \mathrm{~A}$ b).

NOTE: These are holes for two bolts to fix the window frames together.

7. Place the custom-made front chamber frame using bolts (Figures 1B-a, 1B-c) and position the back frame (Figure 1B-b) on the bolts. Place nuts (Figure 1B-d) at the back frame on the bolts.

NOTE: These bolts are used to fix the chambers together against the skinfold and are later used to immobilize the chamber during evaluation under the microscope.

8. Pull the skin $2-3 \mathrm{~mm}$ above the top of the frames and make sure that the transplantable window view area matches the chamber area. Place $23 \mathrm{G}$ needles through the two little suture holes (Figure 1B, black arrow) at the top of the frame. Tighten the nuts on the bolts with a micro screwdriver (Figure 1A-c) and a clamping needle holder to immobilize the nuts. NOTE: Take precautions to ensure that the skin does not turn around the bolts, and do not tighten too much.

9. Replace the needles with sutures, starting at the top, to fix the frames against the skin. Do this for the 5 pairs of suture holes (Figure 1B white arrow) in the frame.

10. Turn the mouse to reveal the backside of the window chamber. Place a thick piece of filler glass with a diameter of $10 \mathrm{~mm}$ and a thickness of $0.55 \mathrm{~mm}$ (Figure 1B-e) and then a standard cover glass of $12 \mathrm{~mm}$ (Figure 1B-f). Secure these with a retaining ring (Figure 1B-g) in the back-chamber area.

NOTE: The filler glass prevents space to open between the fascia and the window at the front side, which is used for imaging.

11. Hydrate the window view area in which the tumor will be inserted by filling a $1 \mathrm{~mL}$ syringe with sterile $0.9 \% \mathrm{NaCl}$. Allow a few drops to fall on this view area. Remove excess saline with a sterile cotton tip. 
12. In the fascia, create a pocket large enough to hold the $1 \mathrm{~mm}^{3}$ tumor fragment (Figure 1A-a) using a $25 \mathrm{G}$ needle with its tip is bent at a $90^{\circ}$ angle (Figure 1A-d). Using a needle holder or Kelly forceps, insert the tumor fragment in the middle of the transplantable window view area in this pocket.

13. Close the window view area with a standard cover glass of $12 \mathrm{~mm}$ and a retaining ring. NOTE: An air bubble can form, but it will disappear within hours.

\section{Pre- and After-care of the Animal}

1. Administer drugs for pain relief (i.e., $0.05 \mathrm{mg} / \mathrm{kg}$ buprenorphine) subcutaneously as approved by your Institutional Animal Care and Use Committee. Allow the mouse to recover from anesthesia under a heating lamp.

2. Place window-bearing mice individually in a cage and in a climate-controlled room set to $32{ }^{\circ} \mathrm{C}$ and above $50 \%$ humidity. Make sure that the water bottles are at environmental temperature before placing them on the cage to prevent leakage. Use cages and enrichment that allow unobstructed movement and access to food and water.

NOTE: As this window chamber is very well tolerated, mice show normal behavior. Individual housing prevents damage to the window chamber caused by other mice, and the high temperature/humidity prevents the cooling down and dehydration of the skinfold.

3. Check the mouse regularly for chewed-off sutures, loose bolts/nuts, or broken cover glass. Immediately repair and replace when this happens. A cover made of light material may be used to protect the window.

\section{Intravital Imaging}

NOTE: In this procedure, a multiphoton microscope and the appropriate control software are used. Here, software packages that were provided with the microscopes are used. In principal, any software package that comes with a microscope and is meant for microscope control and for the capture of images will suite this purpose.

1. Switch on the system: $\mathrm{PC} /$ microscope, power to confocal control unit, laser power, and laser emission. For example, with this software, start the software and initialize the microscope table (Figure 1E-a and 1E-c) by clicking "initialize table." Switch on the system and set it to the mode enabling the PC to control the microscope, xy-table, z-positioning, and image capture.

2. Switch on the fluorescent light (Figure 1E-b). NOTE: This is used to evaluate fluorescence images through the ocular together with bright field.

3. Switch on the custom-made, temperature-controlled platform (Figure $1 \mathbf{C}$-c; described in the Discussion in more detail) set at $37{ }^{\circ} \mathrm{C}$.

4. Switch on the anesthesia unit using isoflurane/ $\mathrm{O}_{2}$ (Figure 1E-d). Mount the anesthesia tube on the microscope stage (Figure 1C-e). Install a clamp on the xy-stage to fix the anesthesia snout piece.

5. Pre-evaluate the animal to establish the stage of tumor vessel development; this depends on the speed of tumor growth, but it can vary between individual mice.

1. Sedate the mouse using inhalation anesthetics (isoflurane/ $\mathrm{O}_{2}$ ) in an anesthesia chamber (see step 2.6).

2. Place the animal on the temperature-controlled platform mounted on the microscope stage; this prevents the cooling down of the animal when it is anesthetized. Make sure the snout of the mouse is located in the anesthesia cone and apply eye ointment if the evaluation will take longer than 5 min.

NOTE: For an evaluation longer than $1 \mathrm{~h}$, reduce the isoflurane flow to $2 \%$.

3. Use the chamber bolts to fix (Figure 1C-b, full arrow) the chamber on a custom-made chamber holder (Figure 1C-a). Screw this holder (Figure 1C-d, dashed arrow) to the heated platform (Figure 1C-c).

NOTE: This combination prevents movement artifacts in the window view area while still allowing the animal to breathe freely.

4. Make sure to clean the glass of the window view area with a cotton tip dipped in water to prevent a blurry image and interference coming from debris on the outside of the glass. Place the platform and mouse on the microscope stage (Figure 1D). NOTE: Take care that the tail does not get stuck between the platform and the microscope stage.

5. Visually check the stage of tumor vessel development using a 10X objective lens, bright-field, and fluorescent light. NOTE: Fluorescent vessels should be in focus and the blood flow should be visible using bright field. When the vasculature is clearly seen, evaluate the stage of tumor vessel development. For instance, determine whether there is early-onset angiogenesis with the growth of tip cells or an already-established vasculature for drug delivery. The same animal can be evaluated again, and as tumor development is a continuous process. Several stages can be observed in a single tumor at the same time.

1. When it is not possible to focus on the vasculature, remove the animal from the stage, let the mouse recover, and place the animal back in a climate-controlled space. Check it again later. When the mouse is ready for evaluation, continue with the process described below.

6. Switch on the confocal lasers and the control panel by clicking "Laser" and "Ctrl panel" in the Configuration tab NOTE: It is recommended to set the laser power low, particularly for the Argon laser, to prevent bleaching, the heating of the tissue, and photodamage. The control panel can be set to personal preferences. For intravital imaging using multiple fluorophores it is recommended to apply the following settings: "smart gain, $100 \mathrm{~V}$ per turn," "smart offset, 10\% per turn," "zoom, medium," "X position, medium," "Y position, medium,"and "Z position, $100 \mu \mathrm{m}$ per turn." Prior to imaging, adjust the offset to minimize the noise and optimize the signal-to-noise ratio. The confocal zoom function allows for a higher magnification without changing the objective lenses. $\mathrm{X}, \mathrm{Y}$, and $\mathrm{Z}$ control is necessary to locate the fields of interest. For intravital microscopy, Z-positioning should be about $100 \mu \mathrm{m}$ per turn as tick tissue is evaluated.

7. Click on "Acquisition" in the Acquire tab and change the setting to: "Acquisition mode XYZ or XYZT," "Format (512x512 or 1024x1024)," and "Speed $(400 \mathrm{~Hz}$ or $200 \mathrm{~Hz})$." Click on "Pinhole" and set it to 1 airy unit. Click on "Bidirectional X".

NOTE: To optimize the images, a balance between laser power, gain, and pinhole should be struck, which is mentioned in the Discussion.

8. When evaluating multiple fluorophores, select "Sequential scan" and "Between frames" to prevent bleed-through, which is mentioned in the Discussion. Choose "Line Average 4" to obtain a good reduction of the noise. 
NOTE: Depending on the intrinsic fluorescence present in the transgenic animal, other fluorescent markers, like dextrans, Hoechst, FITCBSA, fluorescent chemotherapeutics, and/or nanoparticles diluted to the desired concentration in $0.9 \% \mathrm{NaCl}$ can be administered and assessed in the tumor. These are injected through the tail or penile vein.

9. Click on "Experiment" in the Acquire tab and select "New" to make a new data base.

10. Evaluate the animal using, depending on the research question, 10X, 20X, or 40X objective lenses. Through the ocular, find a position to evaluate.

NOTE: It is best to use dry lenses with an NA as high as possible, as dry lenses have a relatively long working distance and do not need immersion fluid. In the Discussion, the use of water-immersion objective lenses is mentioned.

11. Use a fast live scan to find the proper gain and offset to prevent overexposure and to optimize the signal-to-noise ratio. Also, maintain same imaging settings during and between experiments if quantitative comparison is needed.

NOTE: Further considerations for optimal imaging are mentioned in the Discussion. The XYZ position and zoom can be finalized during the live scan using the control panel.

12. To make a Z-stack, select "Z-stack" in the Acquire tab. Make a fast Z-scan using the Z-position in the control panel and set the beginning and end of the scan by clicking "Begin $\mu \mathrm{m}$ " and "End $\mu \mathrm{m}$." Set the Z-step size related to the pinhole size.

13. Capture the images by clicking "start."

NOTE: As intravital microscopy is all about kinetics and therefore dynamic processes are observed, a compromise between the time needed to acquire an image and the speed of the biological processes must be made. In general, the higher the resolution, the more fluorescent channels are scanned and the bigger the scanning field, the more pixels per image. Thicker Z-stacks translate to a longer imaging time. A longer imaging time increases the chance of movement artifacts and may cause damage to the tissue imaged. Also, be aware that scanning certain fluorescent labels causes bleaching and toxicity, which is affected by laser power and the concentration of the accumulated dye.

14. After scanning the Z-stack can be quickly evaluated by clicking "maximal projection". The Z-stack is automatically saved in the data base and can be renamed.

\section{Data Analysis}

1. Depending on the original research question, use one of several software programs, such as Image $\mathrm{J}^{5}$, Matlab ${ }^{11}$, or Amira, for data analysis. Correct analysis is crucial.

\section{Representative Results}

The main attribute of intravital imaging is the longitudinal visualization of cellular processes without invasive intervention. For this, transgenic animals expressing a constitutive or inducible fluorescent marker in cells of interest are used. Figure 2 illustrates the expression of fluorescent labels in eNOStag-green fluorescent protein (GFP) ${ }^{9}$ and Rosa-mTmG mouse lines ${ }^{10}$. The eNOStag-GFP is a mouse line that was produced inhouse using a truncated eNOS gene as a tag for GFP. Importantly, eNOS is highly expressed in endothelial cells, and the GFP signal is clearly seen in th eGolgi and cellular membrane (Figure 2A). Rosa-mTmG mice have a membrane-targeted two-color fluorescence Cre reporter allele. This line expresses mTomato fluorescence in widespread cells and mGFP in Cre recombinase-expressing cells and is vastly used for lineage tracing. As a tumor piece is transplanted from a non-transgenic donor, red fluorescence is predominantly expressed by stromal parts in the tumor (e.g., in the membrane of vascular cells, circulating cells, infiltrated blood cells, and tumor-associated fibroblasts (Figure 2B)).

Vessel formation is tightly regulated, and an excellent model to study this is the developing retina ${ }^{12,13}$. Also, tumor vessel growth is a kinetic process that is similar in principle to retinal vessel development. However, tumor vessels lack organization and, as a tumor is continuously remodeling, so is the tumor-associated vasculature. This can have its advantages when using the tumor as a angiogenic model, as all stages of vessel development can often be found in the same tumor at the same time. These include an endothelial sprouting front growing into an unvascularized part of the tumor (Figure 2C); damaged vessels (Figure 2D); and an established vascular bed (Figure 2E-I) with mature, branched vessels (Figure 2E-II). However, angiogenic endothelial cells can also be found in these areas. When stimulated by angiogenic stimuli, an endothelial cell protrudes filopodia (Figure 2E-III) and can advance into a tip cell, using these filopodia for scanning and directional migration (Figure 2E-IV). This tip cell migrates into the tumor interstitium and is followed by dividing stalk cells. A single endothelial tip cell has several filopodia expanding, retracting and renewing at any time, and can be tracked using intravital microscopy (Figure 2F).

Secondly, intravital microscopy can be used to determine lumen formation at an angiogenic front, blood flow throughout the tumor, and extravasation. Depending on already-present intrinsic fluorescent signals in the animal, different fluorescent compounds can be administered. Blood flow and extravasation can be visualized using Hoechst, fluorescent dextrans, or FITC-BSA. For extended studies on blood flow and particle extravasation, long circulation fluorescently labeled pegylated nanoparticles of $100 \mathrm{~nm}$ can be used. For details on the preparation of these nanoparticles, see a previous publication ${ }^{5}$. The employment of these compounds is demonstrated in Figure 3 . The endothelial tip cells in a Rosa-mTmG mouse can be clearly seen invading the tumor tissue. Functional blood flow is demonstrated by the purple fluorescence of systemically injected nanoparticles in the lumen of vascular tubes (Figure 3A-I). Nanoparticles closely reach endothelial tip cells illustrating the formation of a lumen in the stalk cell area (Figure 3A-II). The delivery of systemically administered chemotherapeutics depends on a functional vasculature in order to reach the tumor interstitium, and as shown in Figure 3B, injectable agents, independent of their size, do not penetrate areas with destroyed vessels, compressed vessels, or vessels with blood stasis.

In most organs, the endothelial lining forms a functional barrier between the blood and underlying tissue, and the passage of the molecules is tightly regulated. Tumor-associated vasculature, however, is known to be leaky, and pore-size cut-off depends greatly on the tumor type ${ }^{14}$ Fluorescent-conjugated dextrans with different sizes can be injected to assess the blood flow, permeability, and extravasation. Hoechst (615 Da) diffuses rapidly into tumor tissue and is taken up by surrounding cells (Figure 3C-I). Shortly after the injection, dextran of $10 \mathrm{KDa}$ (Figure $3 \mathrm{CII}$ ) and $2 \mathrm{MDa}$ (Figure 3C-III) are found in the blood. However, dextran $10 \mathrm{KDa}$ is also seen in the tumor interstitium, indicating the permeability of the endothelial lining for smaller molecules, which is a feature ascribed to tumor vessels. 40 min after injection (Figure 3C-IV), dextran 10 KDa is cleared from the blood stream (Figure $\mathbf{3 C}-\mathbf{V}$ ), and the fluorescent intensity of dextran 2MDa is decreased as well (Figure $\mathbf{3 C}$-VI). However, large dextrans are not found in the tumor interstitium, demonstrating a lack of permeability to large molecules within this time frame. 
Tumor pathophysiology, with its highly proliferating, necrotic, and un-vascularized areas, can be quite informative when using the tumor as an angiogenic model. However, this presents a problem for effective therapy and investigation. The heterogeneity of tumor-associated vasculature causes a heterogeneous distribution of administered drugs, leaving entire areas drug-free ${ }^{15}$. To improve drug delivery, several strategies can be applied $^{15,16,17}$, and therapeutic progression can be examined using this intravital design. The tumor-associated vasculature can be manipulated using vasoactive agents to improve drug delivery ${ }^{17}$. The results of tumor vessel manipulation using tumor necrosis alpha (TNF) as a vasoactive agent in combination with Doxil, the encapsulated liposomal formulation of doxorubicin, as a chemotherapeutic agent are presented in this manuscript ${ }^{5,18,19}$. In contrast to dextrans, pegylated nanoparticles are made to circulate for several hours, if not days, in the blood circulation ${ }^{20}$.

As previously mentioned, the tumor area can be pre-scanned to identify the correct tumor areas depending on the research question. Drug fate can be evaluated in areas with a functional vasculature versus areas with an already damaged vascular bed (data not shown). To investigate the fate of chemotherapeutic agents without cytotoxic interference, nanoparticles with the same composition as the therapeutic agent can be used as a model drug. An eNOStag-GFP animal was treated i.v. with these nanoparticles and imaged $24 \mathrm{~h}$ later. Nanoparticles were still present in the vasculature, with minimal extravasation into the tumor interstitium (Figure 4A-I). However, when nanoparticles were administered in combination with TNF, extravasation was observed from the blood stream into the tumor interstitium (Figure 4A-II), increasing intratumoral drug delivery. By using high-resolution objective lenses, the intracellular localization of a compound can be recognized, as shown here by the nuclear location of Hoechst in green endothelial cells and cells of the tumor interstitium (Figure 4B). Moreover, as many chemotherapeutic agents, like doxorubicin, intercalate with DNA, the localization of these compounds can be evaluated. Doxorubicin has red fluorescent properties, and the nanocarrier can be labeled with, for instance, tetramethylindotricarbocyanine perchlorate (DiD). An eNOStag-GFP animal was injected i.v. with Doxil-DiD in combination with TNF, and images were taken $24 \mathrm{~h}$ after treatment. Doxil-DiD extravasated out of the blood vessels and was taken up by surrounding tumor tissue (Figure 4C-I). A more detailed evaluation of individual cells (Figure 4C-II) showed that the carrier can be found in the cytoplasm, while released doxorubicin was observed in the cellular nucleus.
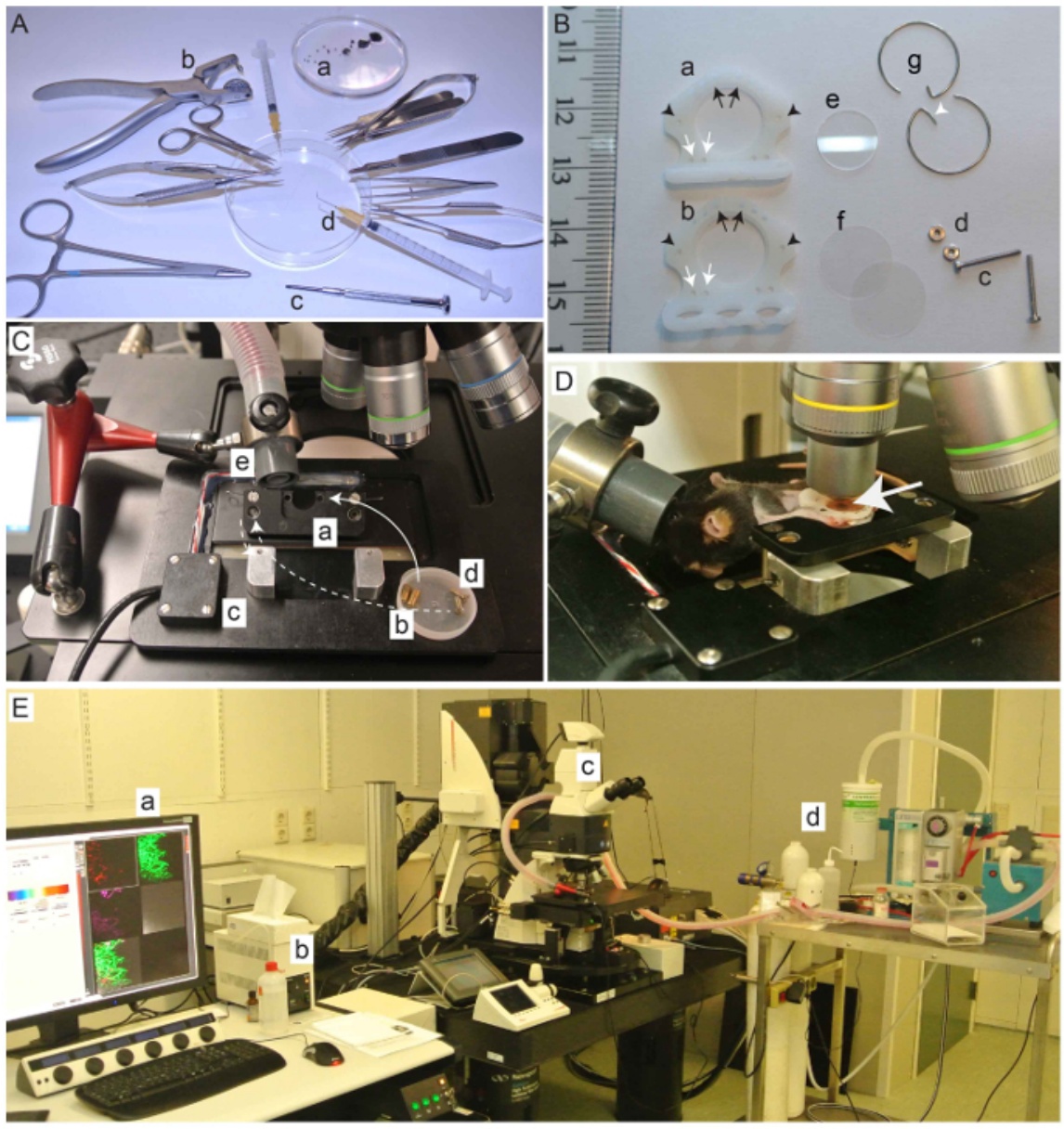

Figure 1: Instruments, dorsal skinfold chamber, and equipment setup needed for the procedure. (A) Tumor fragments (a) and surgical instruments needed for implantation. Non-standard instruments are an ear puncher (b), a micro screw driver (c), and a bent needle (d). (B) Dorsal skinfold window chamber. Front (a) and back (b) window (arrow: holes for sutures; arrowhead: holes for bolts), 2 bolts (c), 2 nuts (d), 1 filler glass (e), 2 cover glasses (f), and 2 retaining rings (g). Also, retaining rings without hooks (white arrowhead) can be used when required. (C) Custom-made chamber holder (a), bolts for the chamber-to-chamber holder (b), temperature-controlled platform (c), bolts to secure the chamber holder to the platform (d), and the holder with the anesthesia tube (e). (D) Animal mounted in the chamber holder on the platform. A B16BL6 tumor (arrow) is visible in the window view area. (E) Equipment needed for the intravital evaluation. Computer with imaging and microscope control software (a), standard fluorescent light (b), and the microscope. A multiphoton confocal was used (c) with an anesthesia unit (d). Please click here to view a larger version of this figure. 

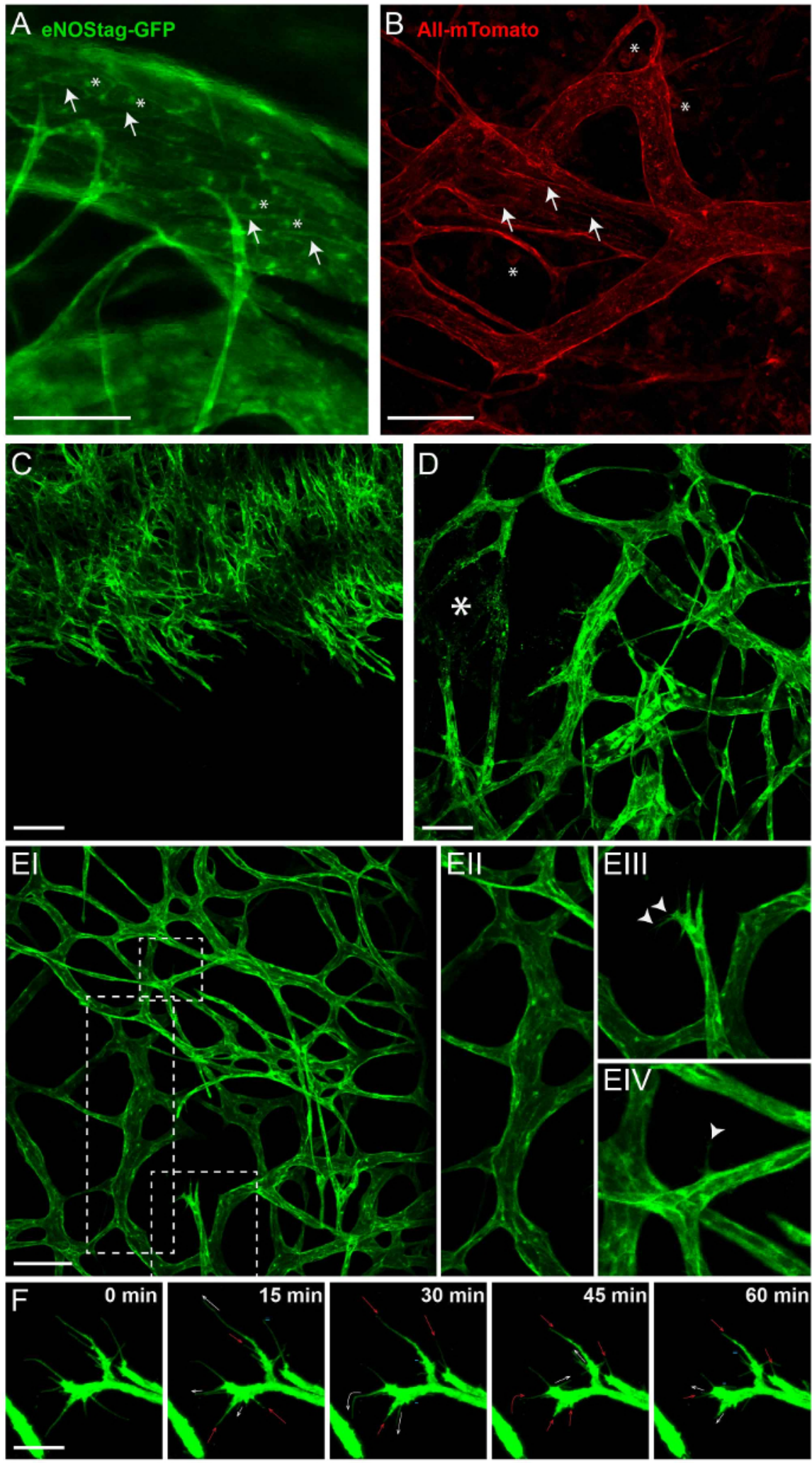

Figure 2: Intrinsic fluorescence of transgenic animals. All images shown here are Z-stack projections between 50 and $70 \mu \mathrm{m}$ thick. (A) In the eNOSGFP-tag line, GFP is expressed in the Golgi (asterisk) and cell membrane (arrow) of endothelial cells. Scale bar $=100 \mu \mathrm{m}$. (B) Intratumoral expression of mTomato in the Rosa-mTmG line is predominantly found in the cell membrane of vascular cells (arrow) and blood cells (asterisk). Scale bar $=100 \mu \mathrm{m}$. (C) A projection of a growing angiogenic front into un-vascularized tumor. Scale bar $=100 \mu \mathrm{m}$. (D) A projection of part of the tumor with established and damaged vessels (asterisk). (E) A projection of established tumor vasculature (EI) showing mature tick vessels (EII); angiogenic endothelial tip cells with filopodia (EIII, arrowhead); and a mature vessel, from which a single endothelial cell extends a filopodium 
(EIV, arrowhead) into the interstitium. Scale bar $=100 \mu \mathrm{m} .(\mathbf{F})$ Movement of the filopodia, followed for $1 \mathrm{~h}$. Every $15 \mathrm{~min}$, a Z-stack was taken; a maximum projection is presented here. The filopodia are extending (white arrow), stagnant (=), retracting (red arrow), or even completely disappearing (-). Scale bar $=25 \mu \mathrm{m}$. Please click here to view a larger version of this figure.
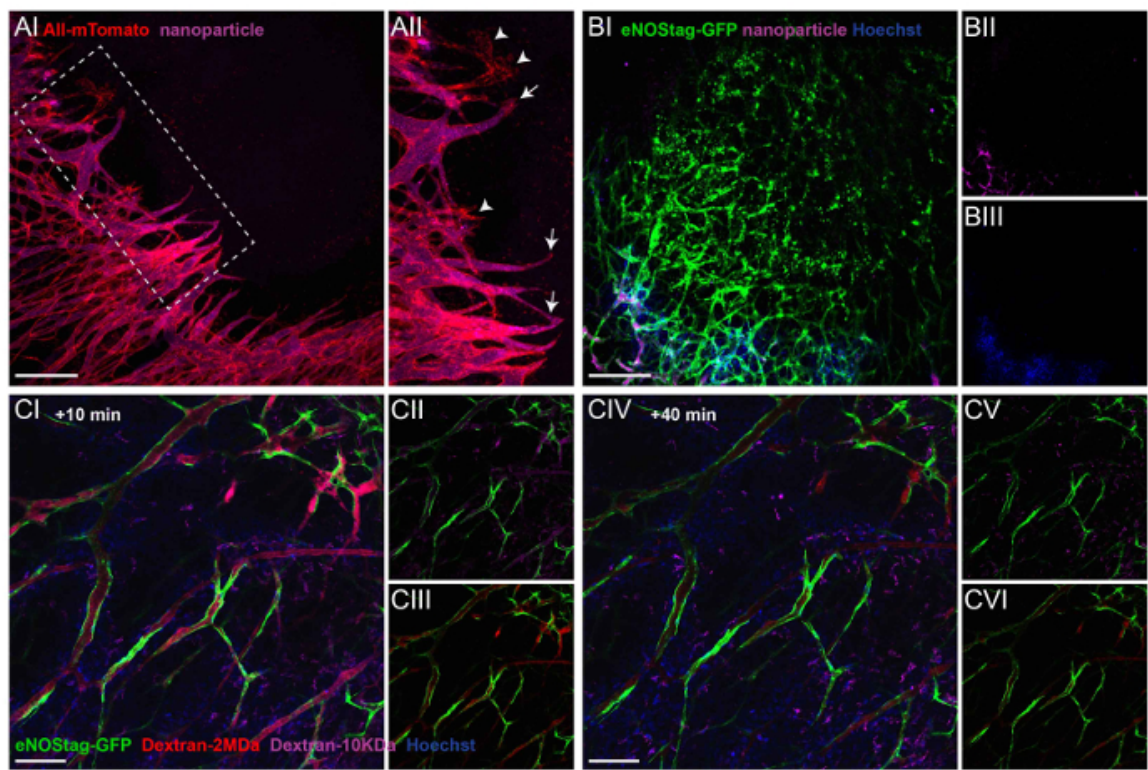

Figure 3: Administration of injectable labels to illustrate extravasation and blood flow. (A) A Rosa mTmG mouse was injected with purple fluorescent nanoparticles, and a Z-stack of an invading tip cell front was taken 10 min later (Al). A projection showing that most endothelial sprouts have a functional lumen (All, arrow). Only rarely can endothelial sprouts be seen without flow (All, arrowhead). Scale bar $=250 \mu \mathrm{m}$. (B) This Z-stack projection shows a destroyed tumor vessel area in an eNOStag-GFP animal prior to treatment (BI). The animal was injected with nanoparticles (purple, BII) and Hoechst (blue, BIII). Nanoparticles and Hoechst do not reach destroyed areas, indicated by granulated cell debris still expressing GFP. Scale bar $=250 \mu \mathrm{m}$. (C) An eNOStag-GFP mouse was injected with two dextrans of different sizes (red = Dextran 2 MDa; purple $=$ dextran $10 \mathrm{KDa}$ ) and Hoechst (blue $=615 \mathrm{Da}$ ), and single-plane images are presented here. 10 min after the injection, presence in the blood and extravasation are seen in the same image. Hoechst extravasates almost immediately out of the blood vessels and is taken up by the surrounding cells $(\mathrm{Cl})$. Dextran $10 \mathrm{KDa}(\mathrm{ClI})$ can be seen in vessels and in the tumor interstitium. Dextran $2 \mathrm{MDa}(\mathrm{CIII})$ can be found in the vessels. $40 \mathrm{~min}$ after injection (CIV), Dextran $10 \mathrm{KDa}$ disappears from the blood (CV), and the fluorescent intensity of Dextran $2 \mathrm{MDa}$ was also diminished $(\mathrm{CVI})$. Scale bar $=100 \mu \mathrm{m}$. Please click here to view a larger version of this figure.
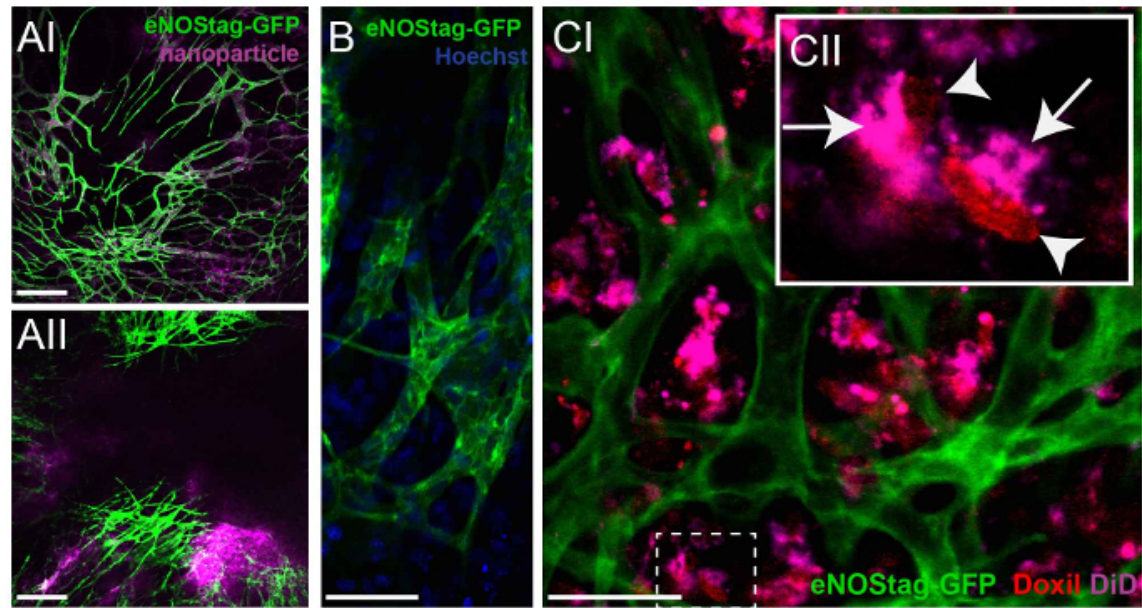

Figure 4: Investigating the fate of chemotherapeutic agents. (A) An eNOStag-GFP animal was treated i.v. with nanoparticles and imaged 24 $\mathrm{h}$ later. Nanoparticles are still present in vessels, with minimal extravasation in the tumor interstitium (Al). When nanoparticles are combined with TNF, extravasation is observed from the blood stream into the tumor interstitium (All). Scale bar $=250 \mu \mathrm{m}$. (B) Using high-resolution lenses, the endothelial cytoplasm (green) and nucleus (blue) of an individual cell can be recognized. Scale bar $=50 \mu \mathrm{m}$. (C) An eNOStag-GFP animal was injected i.v. with Doxil-DiD in combination with TNF, and images were taken $24 \mathrm{~h}$ later. Here, the extravasation of Doxil-DiD out of the vessels into the tumor interstitium is obvious $(\mathrm{Cl})$. A more detailed evaluation of individual cells (CII) shows that the purple carrier can be found in the cytoplasm (arrow), while the released doxorubicin (red) is observed in the cellular nucleus (arrowhead). Scale bar $=50 \mu \mathrm{m}$. Please click here to view a larger version of this figure. 
Discussion

As tumor and tumor vessel development, as well as tumor therapeutic effects, are highly dynamic processes, intravital evaluation is an elegant tool to make a correct assessment of these processes in a time- and spatial-dependent manner. With the increasing availability of live cell markers, the creation of transgenic animals, and the advancement of imaging modalities, intravital imaging is becoming more and more popular. Although histology is still routinely used, and a multitude of markers can be used to identify cells and structures, tissue sectioning has disadvantages when investigating dynamic processes. Tissue dissection is required, providing only static information; fixation affects tissue quality; and slicing damages cellular interactions. Also, when investigating dynamic processes, tissue dissection at different, and often presumed, time-points requires a large quantity of animals. With intravital imaging, the XYZ spatial dimensions and the extra dimension of time can be evaluated in the same animal, making the proper positioning of different players possible in space and time. Therefore, optimal time points are not missed, and the animal number used per experiment is significantly reduced. Second, the time-window established with intravital evaluation can be extrapolated to optimize tissue extraction. Third, the desired stage of vessel growth can be identified intravitally and stained as a wholemount tissue.

The intravital design mentioned in this manuscript uses a combination of transgenic animals expressing a fluorescent label in endothelial cells, a modified dorsal skinfold chamber model, and a dedicated multiphoton-confocal microscope.

Endothelial cells go through a number of stages ${ }^{21,22}$ during angiogenesis, and these stages can often be seen simultaneously in a tumor. Using the eNOSTag-GFP mouse line, individual endothelial cells can be followed, and even filopodia dynamics can be traced. It is therefore a good model to study vessel development intravitally. Depending on the already-present intrinsic labels, injectable fluorescent agents can be used to assess lumen formation, blood flow, extravasation, and blood clearance. The mouse is imaged continuously for up to $5 \mathrm{~h}$. The longterm evaluation of an animal is feasible when several precautions regarding the anesthesia of the animal are taken, which has been described previously ${ }^{23}$. As this research is focused on cancer, tumors tissue or cells were implanted. However, we also experimented with metatarsals and embryonic lungs. However, the growth rate of tissue under study is limiting, as after a couple of weeks, the skin starts to regrow, which can be a problem with slow-growing tumors or tissues.

During the validation phase, the dorsal skinfold window chamber was considerably modified compared to the classical model ${ }^{4}$. The frames are constructed of autoclavable synthetic material and are light and small, with a large window-view area. The hook of the retaining ring (Figure $1 \mathrm{Bg}$, white arrowhead) is only used for the easy removal of the ring. Retainer rings without hooks can be used when these interfere with imaging. The skin is not stretched too much, loosening does not occur in this model, and infections are only rarely seen. These modifications reduce animal discomfort and refine the animal procedure significantly. Also, the metal parts can be easily removed when imaging the tumor using $\mathrm{MRI}^{24}$.

Any microscope can be adopted to intravitally image dorsal skinfold chambers. The main requirement is the available space between the microscope stage and the objective lens. An important aspect that affects imaging is motion. To prevent motion artifacts, a platform that fits in the microscope table (after the removal of all inserts) and supports the mouse should be used. It is not necessary to restrain the mouse when it is under anesthesia, and it is better to let the mouse breath freely. However, the window is bolted onto the platform, giving optimal fixation of the field of view (i.e., the tumor is visible through the window chamber). The platform is heated using an electronic heating pad, as used for animal heating. This platform was made in-house, and specifics can be obtained upon request. A high-resolution objective lens is a necessity when evaluating intracellular processes and makes it possible to discriminate between the cytoplasm and the nucleus. The use of a tapered lens with a good optical resolution (high NA) but a relatively long working distance (preferably $2 \mathrm{~mm}$ or above) is recommended. The limitation in imaging is the penetration depth and the fluorescent intensity of the labels. Furthermore, photobleaching, phototoxicity, and saturation must be avoided during imaging.

Here, an integrated confocal-multiphoton and a confocal microscope were used. The multiphoton is upright, while the confocal is an inverted microscope. The advantage of an upright microscope is the easy use of water-immersion lenses. These lenses have a high NA and a long working distance, even at a high (e.g., 20 or 40X) magnification. Specifically, it is recommended to obtain a $20 \mathrm{X}$ NA 1.0 water-immersion lens, which is sold by several companies. Be aware that, when immersion lenses are used, the objective lens and immersion fluid need to be heated to $37^{\circ} \mathrm{C}$. For the best images, it is recommended to use optimal confocal settings (i.e., pinhole at 1 airy unit, laser power as low as possible, gain not too high (especially if the gain introduces noise), line speed at maximum, and no averaging of scans). Overexposure, saturation, and difference in intensities between images compromise data quality. It is recommended to prevent saturation and overexposure. This can be circumvented by acquiring images at different gain settings. Changing the gain is the easiest way to change the image brightness. If needed, the laser power can be adjusted. To standardize the image quality, slides with a fixed fluorescence intensity can be used. One must keep in mind that even when the microscope is set optimally, image quality is determined to a great extent by the quality of the window chamber and tissue

When scanning multiple fluorescent markers simultaneously, bleed-through, in which one fluorophore is detected in the channel of another one, must be avoided. Avoid bleed-through by using a combination of fluorophores with minimal overlapping spectra and sequential scanning between frames. The images presented here were scanned using 3 sequential scans (Track 1: GFP, DID or fluor647 with the 488 and 633 laser; track 2: rhodamine, Doxil, or mTomato with the 543 laser; and track 3: Hoechst with the 405 laser).

The possibility of evaluating several stages of tumor vessel development, tip cell dynamics, lumen formation, extravasation, and vascular damage is shown here. Using the eNOStag-GFP line, tumor areas with a destroyed vascular bed are easily detected by granulated cellular leftovers still expressing GFP. No injectable agent was detected in these areas, indicating the lack of flow. This means that administered chemotherapeutic agents will not reach these areas either. However, vessel destruction can also be a therapeutic result. Pre-evaluation of the tumor, to check for pre-treatment vessel destruction, is a prerequisite for the accurate therapeutic evaluation and can be easily performed using this experimental design.

There is a plethora of possibilities for which intravital microscopy can be used, and detailed discussion is beyond the scope of this publication. To give a brief insight, possible applications include studies on the accumulation of chemotherapeutics in tumor tissue, the development of vessels (e.g., the number of vessels, branches, and intersections) and blood flow, cell-cell interactions, cell targeting, and intracellular processing, as well 
as examples mentioned above and shown here. As analysis is based on fluorescence, be aware of intensity fluctuations due to the quality of the window or tissue. Also, as tumor tissue is relatively thick, fluorescence from above or below the plane of view has an impact on the signal in the image. It is best to combine the quantitative image analysis with objective measurements. For instance, if interested in drug concentrations, remove the tumor tissue from the window after intravital microscopy and determine the drug content using with HPLC to compare with the confocal images.

The evaluation of tumor response can be performed as well using this model, but with some precautions. One must realize that tumors also grow inwards, into the skin. 3D measurements can be made if the penetration is deep enough to cover the entire tumor. In such a case, farred dyes should be used. The window chamber as described here deals with tumors in a skin environment, and it is important to point out that the window maintains a temperature slightly lower than the normal mouse body temperature. Therefore, it is best to study tumors in the right micro environmental setting to confirm intravital studies with the dorsal skinfold window chamber. Importantly, the dorsal skinfold chamber offers insight into the kinetics of processes and mechanisms, providing an instrumental basis for the improvement of therapy. Also, extra information on the biodistribution and pharmacokinetics can be obtained. Classically, these biodistribution studies are performed by treating tumor-bearing animals and dissecting tumors/organs to measure drug uptake. Using this intravital model, the intratumoral location of a drug (i.e., intravascular, intratumoral, intracellular, or nuclear) can be provided ${ }^{5,25,26}$. Not only is the location of the drug revealed, but also the time window-of-opportunity for the most optimal uptake .

Using the experimental design described in this article, a wide range of parameters can be evaluated in a temporal and spatial setting. Specifically, here we show that intravital microscopy provides important insights into the dynamics of tumor vessel development and therapeutic response.

\section{Disclosures}

The authors have nothing to disclose.

\section{Acknowledgements}

We would like to thank Rien van Haperen and Rini de Crom for their development and donation of the eNOSTag-GFP line, Joost A.P. Rens for his technical assistance with the animal work, and the animal caretakers for their help. The microscopy facilities used are part of the Erasmus Optical Imaging Center, and we would like to thank the staff of the OIC for their service. This study was supported by grant DDHK $2000-2224$ from the Dutch Cancer Society, "Vereniging Trustfonds" Erasmus University Rotterdam, and "Stichting Erasmus Heelkundig Kankeronderzoek," and we thank the members of the committees for their generous donation.

\section{References}

1. Massoud, T. F., \& Gambhir, S. S. Molecular imaging in living subjects: seeing fundamental biological processes in a new light. Genes Dev. 17, $545-580$ (2003).

2. Ellenbroek, S. I., \& van Rheenen, J. Imaging hallmarks of cancer in living mice. Nat Rev Cancer. 14, 406-418 (2014).

3. Brown, E., Munn, L. L., Fukumura, D., \& Jain, R. K. In vivo imaging of tumors. Cold Spring Harb Protoc. 2010(7), pdb prot5452 (2010).

4. Palmer, G. M. et al. In vivo optical molecular imaging and analysis in mice using dorsal window chamber models applied to hypoxia, vasculature and fluorescent reporters. Nat. Protocols. 6, 1355-1366 (2011).

5. Seynhaeve, A. L. et al. Tumor necrosis factor alpha mediates homogeneous distribution of liposomes in murine melanoma that contributes to a better tumor response. Cancer Res. 67, 9455-9462 (2007).

6. Schiffelers, R. M. et al. Ligand-targeted liposomes directed against pathological vasculature. J Liposome Res. 12, 129-135 (2002).

7. Straetemans, T. et al. T-cell receptor gene therapy in human melanoma-bearing immune-deficient mice: human but not mouse T cells recapitulate outcome of clinical studies. Hum Gene Ther. 23, 187-201 (2012).

8. Pittet, M. J., \& Weissleder, R. Intravital imaging. Cell. 147, 983-991 (2011).

9. van Haperen, R. et al. Functional expression of endothelial nitric oxide synthase fused to green fluorescent protein in transgenic mice. Am $J$ Pathol. 163, 1677-1686 (2003).

10. Muzumdar, M. D., Tasic, B., Miyamichi, K., Li, L., \& Luo, L. A global double-fluorescent Cre reporter mouse. Genesis. 45, 593-605 (2007).

11. Manzoor, A. A. et al. Overcoming limitations in nanoparticle drug delivery: triggered, intravascular release to improve drug penetration into tumors. Cancer Res. 72, 5566-5575 (2012).

12. Pitulescu, M. E., Schmidt, I., Benedito, R., \& Adams, R. H. Inducible gene targeting in the neonatal vasculature and analysis of retinal angiogenesis in mice. Nat Protoc. 5, 1518-1534 (2010).

13. Tual-Chalot, S., Allinson, K. R., Fruttiger, M., \& Arthur, H. M. Whole mount immunofluorescent staining of the neonatal mouse retina to investigate angiogenesis in vivo. J Vis Exp., e50546 (2013).

14. Hobbs, S. K. et al. Regulation of transport pathways in tumor vessels: role of tumor type and microenvironment. Proc Natl Acad Sci U S A. 95, 4607-4612 (1998).

15. Minchinton, A. I., \& Tannock, I. F. Drug penetration in solid tumours. Nat Rev Cancer. 6, 583-592 (2006).

16. Koning, G. A., Eggermont, A. M., Lindner, L. H., \& ten Hagen, T. L. Hyperthermia and thermosensitive liposomes for improved delivery of chemotherapeutic drugs to solid tumors. Pharm Res. 27, 1750-1754 (2010).

17. Seynhaeve, A. L., Eggermont, A. M., \& ten Hagen, T. L. TNF and manipulation of the tumor cell-stromal interface: "ways to make chemotherapy effective". Front Biosci. 13, 3034-3045 [pii] (2008).

18. Brouckaert, P. et al. Tumor necrosis factor-alpha augmented tumor response in B16BL6 melanoma-bearing mice treated with stealth liposomal doxorubicin (Doxil) correlates with altered Doxil pharmacokinetics. Int J Cancer. 109, $442-448$ (2004). 
19. Hoving, S., Seynhaeve, A. L., van Tiel, S. T., Eggermont, A. M., \& ten Hagen, T. L. Addition of low-dose tumor necrosis factor-alpha to systemic treatment with STEALTH liposomal doxorubicin (Doxil) improved anti-tumor activity in osteosarcoma-bearing rats. Anticancer Drugs. 16, 667-674 (2005).

20. Gabizon, A. et al. Prolonged circulation time and enhanced accumulation in malignant exudates of doxorubicin encapsulated in polyethyleneglycol coated liposomes. Cancer Res. 54, 987-992 (1994).

21. Bergers, G., \& Benjamin, L. E. Tumorigenesis and the angiogenic switch. Nat Rev Cancer. 3, 401-410 (2003).

22. Eilken, H. M., \& Adams, R. H. Dynamics of endothelial cell behavior in sprouting angiogenesis. Curr Opin Cell Biol. 22, 617-625 (2010).

23. Nakasone, E. S., Askautrud, H. A., \& Egeblad, M. Live imaging of drug responses in the tumor microenvironment in mouse models of breast cancer. J Vis Exp., e50088 (2013).

24. van Vliet, M. et al. MR angiography of tumor-related vasculature: from the clinic to the micro-environment. Radiographics. 25 Suppl 1 , S85-97; discussion S97-88 (2005).

25. Dicheva, B. M. et al. Cationic thermosensitive liposomes: a novel dual targeted heat-triggered drug delivery approach for endothelial and tumor cells. Nano Lett. 13, 2324-2331 (2013).

26. Li, L. et al. Improved intratumoral nanoparticle extravasation and penetration by mild hyperthermia. J Control Release. 167, 130-137 (2013). 